\title{
Large reservoirs of turbulent diffuse gas around high-z starburst galaxies
}

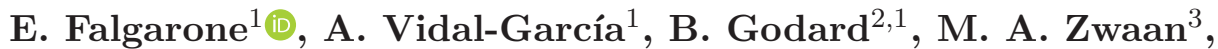

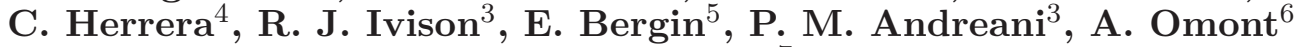 \\ and F. Walter ${ }^{7}$ \\ ${ }^{1}$ Laboratoire de Physique de l'ENS, ENS, Université PSL, CNRS, Sorbonne Université, \\ Université de Paris, 24 rue Lhomond, 75005 Paris, France \\ email: edith.falgarone@ens.fr \\ ${ }^{2}$ LERMA, Observatoire de Paris, CNRS, 61 avenue de l'Observatoire, 75014 Paris, France \\ ${ }^{3}$ European Southern Observatory, Karl-Schwarzschild-Strasse 2, 85748 Garching, Germany \\ ${ }^{4}$ IRAM, 300 rue de la Piscine, Domaine universitaire, 38406 Saint Martin d'Hères, France \\ ${ }^{5}$ University of Michigan, 311 West Hall, 1085 S. University Ave, Ann Arbor, MI 48109, USA \\ ${ }^{6}$ UMPC Université Paris 6 \& Institut d'Astrophysique de Paris, CNRS, 75014 Paris, France \\ ${ }^{7}$ Max Planck Institute für Astronomie, Heidelberg, Germany
}

\begin{abstract}
Starburst galaxies at $z \sim 2-4$ are among the most intensely star-forming galaxies in the universe. The way they accrete their gas to form stars at such high rates is still a controversial issue. ALMA has detected the $\mathrm{CH}^{+}(J=1-0)$ line in emission and/or absorption in all the gravitationally lensed starburst galaxies targeted so far at $z \sim 3$. Its unique spectroscopic and chemical properties enable $\mathrm{CH}^{+}$to highlight the sites of most intense dissipation of mechanical energy. The absorption lines reveal highly turbulent, massive reservoirs of low-density molecular gas. The broad emission lines, arising in myriad UV-irradiated molecular shocks, reveal powerful galactic winds. The $\mathrm{CH}^{+}$lines therefore probe the fate of prodigious energy releases, due to infall and/or outflows, and primarily stored in turbulence before being radiated by cool molecular gas. The turbulent reservoirs act as mass and energy buffers over the duration of the starburst phase.
\end{abstract}

Keywords. galaxies: starburst, galaxies: high-redshift, galaxies: ISM, galaxies: formation, intergalactic medium, turbulence, shock waves

\section{The power of absorption spectroscopy}

In cosmological simulations, the growth of galaxies in the early universe results from the accretion of fresh gas in virialised dark matter halos, modulo ejection of matter by stars and AGNs (Madau \& Dickinson 2014). While ejection is observed in ubiquitous ionised and neutral galactic winds (Veilleux et al. 2005) and contributes to the self-regulation of cosmic star formation (Schaye et al. 2015), evidence for accretion has remained elusive. The dust continuum emission of starburst galaxies at high-redshift makes them extremely bright sub-millimetre sources that provide unique background sources to probe their large scale environment with highly sensitive absorption spectroscopy in the sub-millimetre domain, just as quasars (QSO) do in the visible domain.

The sub-millimetre domain happens to be that of the fundamental transitions of light hydrides, that are the very first steps of chemistry in space, linking hydrogen with heavy elements (Gerin et al. 2016). Among all hydrides, $\mathrm{CH}^{+}$, one of the three first molecular species ever detected in space (Douglas \& Herzberg 1941), has unique chemical and 

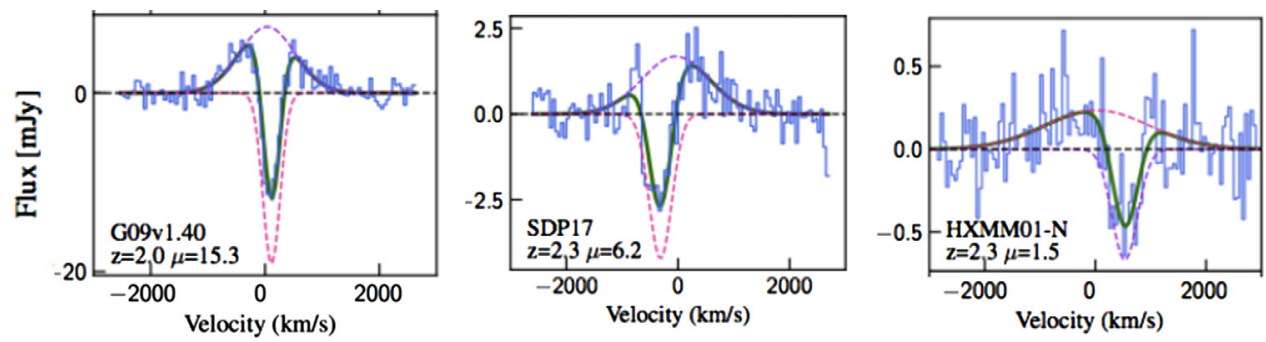

Figure 1. A subset of ALMA continuum-subtracted $\mathrm{CH}^{+}(J=1-0)$ spectra of starburst galaxies illustrating the three kinds of spectra found in the observations. Left: Broad emission and narrow absorption centered at about the same velocity. Middle: P-Cygni profile with broad emission and blue-shifted absorption. Right: Inverse P-Cygni profile with broad emission and red-shifted absorption. The galaxy name, redshift and lens magnification are given in the lower left corner. Note the factor of 50 between the emission line intensity of the brightest and weakest source.

spectroscopic properties. $\mathrm{CH}^{+}$has both a highly endoenergic formation $\left(E_{\mathrm{form}} \sim 0.4 \mathrm{eV}\right)$ and a fast destruction rate, by collisions with $\mathrm{H}$ and $\mathrm{H}_{2}$ : in low-density and weakly irradiated gas, a warm chemistry activated by dissipation of turbulence in shocks and/or intense velocity shears is able to overcome its fast destruction (Godard et al. 2014 and references therein). Once formed, its lifetime is so short, $t \sim 1 \mathrm{yr}$, even in low-density gas, that, unlike the $\mathrm{CO}$ molecule, $\mathrm{CH}^{+}$is always observed where it forms, i.e. it is not transported. Moreover, $\mathrm{CH}^{+}$being a light hydride with high dipole moment, the critical density $\left(\sim 10^{7} \mathrm{~cm}^{-3}\right)$ of its $J=1-0$ transition is almost $10^{5} \times$ larger than that of $\mathrm{CO}(J=1-0)$, so the line appears in absorption in low-density gas $\left(n_{\mathrm{H}}<10^{3} \mathrm{~cm}^{-3}\right)$, and its opacity is a direct measure of the $\mathrm{CH}^{+}$abundance. This abundance in turn provides the turbulent dissipation rate, in the scenario where the supra-thermal energy required for its formation is provided by turbulent dissipation (Godard et al. 2014). In emission, the $\mathrm{CH}^{+}(J=1-0)$ line is detected only in dense and UV-illuminated gas, either photon-dominated regions or UV-irradiated molecular shocks because, unlike most molecules, the abundance of $\mathrm{CH}^{+}$is enhanced in intense UV fields (Godard et al. 2019). The $\mathrm{CH}^{+}$absorption and emission lines therefore both highlight the sites of dissipation of mechanical energy. By doing so, they reveal not only gas components that have never been detected before but also new trails of the gravitational potential energy.

\section{High detection rate of $\mathrm{CH}^{+}$(1-0) lines in high- $z$ starburst galaxies}

With ALMA, the $\mathrm{CH}^{+}(J=1-0)$ line has been detected in all the 18 lensed submillimetre-selected galaxies (SMGs) targeted so far at redshifts $z=1.7-4.2$ (Falgarone et al. 2017, Falgarone et al., in prep.). These are lensed starburst galaxies discovered by Herschel (Eales et al. 2010; Oliver et al. 2010) and SCUBA-2 (Ivison et al., 2010). $\mathrm{CH}^{+}$absorption lines are found in 15 of the 18 sources observed. The absorptions are deep (opacities 0.25-1.2) and broad (average FWHM $\sim 400 \mathrm{~km} \mathrm{~s}^{-1}$ ), blue- or red-shifted with respect to the SMG redshift at almost the same occurrence, (i.e. the average offset is $\sim 60 \mathrm{~km} \mathrm{~s}^{-1}$ ) with a large scatter (i.e. the variance of the offset velocities is $300 \mathrm{~km} \mathrm{~s}^{-1}$ ). The emission lines, when fully visible, are extremely broad (FWHM $\sim 1500 \mathrm{~km} \mathrm{~s}^{-1}$ ) and are understood as originating in myriad dense shocks presumably powered by hot galactic winds penetrating the circum-galactic medium (CGM). In Fig. 1, three spectra illustrate the variety of the overall $\mathrm{CH}^{+}$lineshapes. In several cases, the absorption centroid is only slightly shifted compared to that of the emission (left panel) but in other cases, the whole profile can be either P-Cygni, revealing outflowing material (central panel) or inverse P-Cygni, revealing infalling gas (right panel). 
Since blue- (resp. red-) shifted absorption against a background continuum source unambiguously traces outflowing (resp. infalling) gas with respect to the background source, the similar occurrence of blue- and red-shifted absorptions suggests that absorption lines sample gas that is as often infalling towards the starburst galaxy, as outflowing from it. We ascribe this diversity to large scale turbulence of the gas sampled randomly by the line-of-sight towards the SMGs, the width of the absorption lines providing the gas turbulent velocity dispersion. This similar occurrence of blue- and red-shifted absorption lines can also be seen as a manifestation of the co-existence of outflows and infall, with time-dependent domination of either one (i.e. bursty accretion followed by bursty outflows). The sign of the absorption velocity offset may also depend on the orientation of the outflow and infall directions (if any) with respect to the line-of-sight (Zabl et al. 2019). Nonetheless, turbulence resulting from a fluid instability unavoidable in large-scale cosmic flows, it has to be ubiquitous in those systems.

\section{Starburst CGM properties}

It is well known that absorption lines alone cannot provide the extent of the absorbing medium along the line-of-sight. The method followed to determine the size and mass of the turbulent gas reservoirs (TR) traced by the $\mathrm{CH}^{+}$absorption is detailed in Falgarone et al., (2017). It involves the following steps: (1) we infer that the TR lifetime is of the order of the duration of the starburst phase, $t_{\mathrm{SB}}$, given the high occurrence of absorption detections, (2) the $\mathrm{CH}^{+}$abundance, proportional to the turbulent energy transfer rate, depends on the gas velocity dispersion $\sigma_{\mathrm{TR}}$ (inferred from the absorption linewidth $\left.\Delta v_{\mathrm{abs}}=2.35 \sigma_{\mathrm{TR}}\right)$ and the unknown size $r_{\mathrm{TR}}$, causing a degeneracy between the $\mathrm{CH}^{+}$ abundance and $r_{\mathrm{TR}},(3)$ we break this degeneracy with the finding that, in the five cases where the stelllar mass of the starburst is known, the radius at which the escape velocity of the galaxy surrounded by its massive CGM is equal to the mean turbulent velocity is approximately $\sim \Delta v_{\mathrm{abs}} t_{\mathrm{SB}}$, with $t_{\mathrm{SB}} \sim 50 \mathrm{Myr}$, and in spite of very different stellar masses and gas velocity dispersions. We therefore adopt the estimate $r_{\mathrm{TR}} \sim \Delta v_{\mathrm{abs}} t_{\mathrm{SB}}$, meaning that the TR is virialized in the potential well of the galaxy and that its lifetime is the dynamical timescale of its turbulence, $t_{\mathrm{dyn}} \sim r_{\mathrm{TR}} / \sigma_{\mathrm{TR}}$. A validation of these unrelated assumptions can only be achieved by direct imaging of the CGM.

Once the size is known, the gas mass is directly inferred from the absorption line and the $\mathrm{CH}^{+}$abundance. Massive, $M_{\mathrm{TR}}$ up to a few $10^{10} \mathrm{M}_{\odot}$, and highly turbulent reservoirs, extending far outside $\left(r_{\mathrm{TR}}>10 \mathrm{kpc}\right)$ the compact starburst cores of radii $\approx 1 \mathrm{kpc}$ are therefore unveiled. The resulting gas fraction within tens of $\mathrm{kpc}$ therefore approaches $f_{\text {gas }} \sim 0.5$. These large-scale gaseous halos are likely multi-phasic, comprising the cool $(T \sim 100 \mathrm{~K})$, low-density and turbulent gas in which $\mathrm{CH}^{+}$forms, probably embedded in a warmer and more dilute gas.

\section{Link of the turbulent luminosity and the star formation rate (SFR)}

A remarkable finding, given the many assumptions discussed above and the uncertainties on the SFRs, is that the turbulent luminosity of 11 of the TRs, $L_{\text {turb }}=$ $\frac{1}{2} M_{\mathrm{TR}} \sigma_{\mathrm{TR}}^{2} / t_{\mathrm{dyn}}$ is found to be proportional to the SFR of the galaxies (Fig. 2). The SFRs are derived from the total FIR luminosity (Kennicutt 1998) and could be overestimated by large factors if AGNs contribute, and if the stellar initial mass function (IMF) is not a Salpeter IMF (Zhang et al. 2018). This linear relation, however, provides a key insight to the dynamics of the whole CGM.

The broad $\mathrm{CH}^{+}$emission lines arise in myriad UV-irradiated molecular shocks at $v_{\mathrm{sh}}<50 \mathrm{~km} \mathrm{~s}^{-1}$. Their width traces the velocity dispersion of the shocks themselves, likely driven by galactic-scale high-velocity winds. The shocks are thought to form at the 


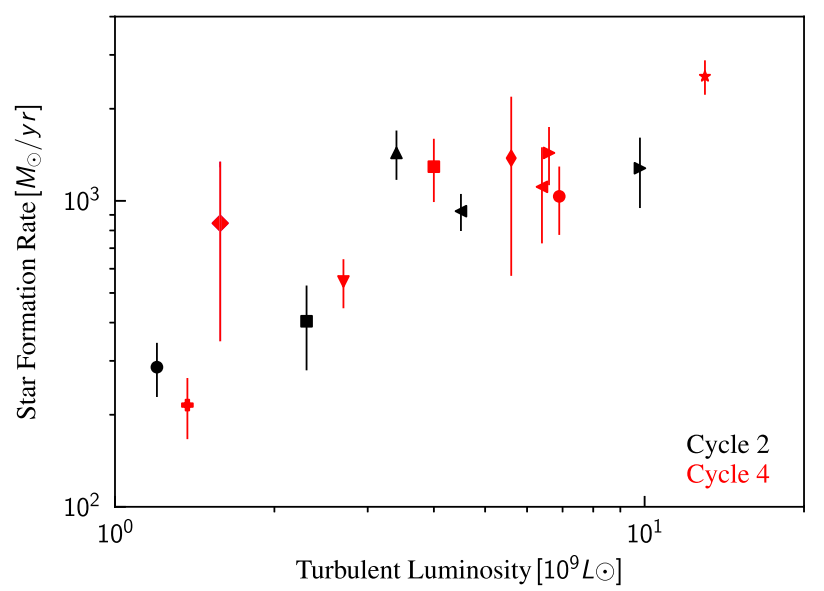

Figure 2. Star formation rates (SFR) of starburst galaxies at $1.7<z<4.2$ vs. the turbulent luminosity of their massive reservoirs of diffuse gas unveiled by $\mathrm{CH}^{+}(1-0)$ absorption. Nominal errors bars on $L_{\text {turb }}$ are less than a factor of 2 , those on the SFRs may be larger than displayed due to possible AGN contributions to the FIR luminosity and top-heavy initial mass function (see text).

interface of the wind and the CGM. The $\mathrm{CH}^{+}$emission linewidth, close to $1500 \mathrm{~km} \mathrm{~s}^{-1}$ in all the cases where it is fully detected, provides the velocity of the outflowing material, $v_{\text {out }} \sim 700 \mathrm{~km} \mathrm{~s}^{-1}$. The outflows trigger large-scale turbulence (and phase transition) in the CGM so that the TR turbulent luminosity is fed by these outflows, $L_{\text {turb }} \propto \dot{M}_{\text {out }} v_{\text {out }}^{2}$. The mass outflow rates, $\dot{M}_{\text {out }}$, are found to be too small to offset the SFRs and we infer from this imbalance that inflows have to contribute to the mass feeding of the TRs to explain their long lifetime around such active starburst galaxies.

Indeed, $\mathrm{CH}^{+}$absorption lines in the direction of SMMJ02399, a small group of galaxies comprising a broad absorption line (BAL) QSO and a SMG (Ivison et al. 2010), are red-shifted by $\sim 500 \mathrm{~km} \mathrm{~s}^{-1}$, indicating an inflow towards the SMG of this magnitude. The size of the TR around these galaxies is inferred to be $r_{\mathrm{TR}} \sim 20 \mathrm{kpc}\left(t_{\mathrm{SB}} / 50 \mathrm{Myr}\right)$. The $\mathrm{CH}^{+}$emission lines have FWZI $\sim 2500 \mathrm{~km} \mathrm{~s}^{-1}$. A most extended (>80 kpc) Ly $\alpha$ nebula has been imaged with the Keck/KCWI in that field (Li et al. 2019) with remarkable properties: the Ly $\alpha$ lines in the direction of the SMG and the BAL QSO are all extremely broad, with FWZI $\sim 2500 \mathrm{~km} \mathrm{~s}^{-1}$. They are asymmetric, with a red-shifted side much weaker than the blue-shifted side, a characteristic of infall (Verhamme et al. 2006). Interestingly, the velocity coverage of the $\mathrm{CH}^{+}$absorptions in this field is the same as that of the red side of the Ly $\alpha$ lines. The comparison of the $\mathrm{CH}^{+}$absorption and emission lines with those of $\operatorname{Ly} \alpha$ led to the conclusion that the front part of the Ly $\alpha$ nebula (and possibly the whole nebula) is inflowing towards the galaxies (Falgarone et al. 2020). It is therefore a multi-phasic medium in which the warm gas (partially or fully ionized) at $10^{4} \mathrm{~K}$ that emits Ly $\alpha$ is mixed with much cooler and denser gas in which $\mathrm{CH}^{+}$ forms because the Ly $\alpha$ emission and the CGM that absorbs $\mathrm{CH}^{+}$have similar spatial extent and velocity coverage. Lastly, the similarity of the FWZI of the $\mathrm{Ly} \alpha$ and $\mathrm{CH}^{+}$ emission lines suggests that some contribution to the Ly $\alpha$ line comes from self-irradiated molecular shocks (Lehmann et al., these proceedings).

\section{The emerging picture}

This is work in progress. Although the above quantitative estimates have been achieved so far with only a handful of galaxies, a coherent picture may be drawn that relies on the fact that $\mathrm{CH}^{+}$is a unique tracer of dissipative processes. The $\mathrm{CH}^{+}$lines, in absorption, 
unveil massive reservoirs of diffuse molecular gas around starburst galaxies at $z \sim 2-4$ that are long-lived, multi-phasic, and extend up to $40 \mathrm{kpc}$ from the starburst cores. In emission, the $\mathrm{CH}^{+}$lines trace molecular shocks with prodigious velocity dispersions that are likely driven by galactic winds. Gas accretion is required to sustain the long lifetime of these turbulent massive reservoirs. The origin of this accretion is unknown: it might be due to tidal streams in mergers or cold streams of pristine gas. Turbulence driven by the galactic winds likely processes the circumgalactic matter and allows the outflowing gas to be recycled, mitigating the negative feedback. Turbulence kinematically heats the CGM, making it a long lived buffer of mass and energy.

\section{References}

Douglas, A. E. \& Herzberg, G. 1941, ApJ, 94, 381

Eales, S., Dunne, L., Clements, D., et al. 2010, PASP, 122, 499

Falgarone, E., Zwaan, M.A., Godard, B., et al. 2017, Nature, 548, 430

Gerin, M., Neufeld, D. A., \& Goicoechea, J. R.. 2016, ARAA, 54, 181

Godard, B., Falgarone, E., \& Pineau des Forêts, G. 2014, A\&6A, 570, A27

Godard, B., Pineau des Forêts, G. Lesaffre, P., et al. 2019, A\&A, 622, A100

Ivison, R. J., Swinbank, A. M., Swinyard, B., et al. 2010, A\&广A, 518, L35

Kennicutt, Jr Robert C. 1998, ApJ, 498, 541

Li, Q., Cai, Z., Prochaska, J. X., et al. 2019, ApJ, 875, 130

Madau, P. \& Dickinson, M. 2014, ARAA, 52, 415

Oliver, S. J., Wang, L., Smith, A. J., et al. 2010, A\&BA, 518, L21

Schaye, J., Crain, R. A., Bower R. G., et al. 2015, MNRAS, 446, 521

Veilleux, S., Cecil, G., \& Bland-Hawthorn, J. 2005, ARAA, 43, 769

Verhamme, A., Schaerer, D., \& Maselli, A. 2006, A\&A 460, 397

Zabl, J., Bouché, N. F., Schroetter, L., et al. 2019, MNRAS, 485, 1961

Zhang, Z.-Y., Ivison, R. J., George, R. D., et al. 2018, MNRAS, 481, 59 